\title{
Estrategias docentes para la formación de alumnos extranjeros en la Universidad de Burgos
}

\author{
Ángel RoDRÍGUEZ SAIZ \\ Universidad de Burgos \\ arsaizmc@ubu.es
Sara GuTIÉRREZ-GONZÁLEZ
Universidad de Burgos
sggonzalez@ubu.es

Recibido: 28/11/2012

Aceptado: 23/01/2013

\begin{abstract}
Resumen
Son muchos los alumnos extranjeros que eligen nuestro país para perfeccionar su formación universitaria, realizando intercambios con Universidades Españolas. La Universidad de Burgos recibe anualmente alumnos de diferentes países, entre los que destacan los procedentes de los Estados Unidos Mexicanos. Los acuerdos entre la Universidad de Burgos y las Universidad Mexicanas de los Estados de Baja California, San Luis Potosí, Chihuahua o México D.F. han posibilitado que muchos alumnos de las Facultades de Arquitectura cursen asignaturas de las Titulaciones de Ingeniería de Edificación e Ingeniería de Caminos Canales y Puertos. En este artículo se desarrolla una estrategia formativa que los profesores de la asignatura de Prevención y Seguridad en la Construcción, de la Titulación de Ingeniería de Edificación, han puesto en práctica, con el objetivo me perfeccionar el conocimiento de esta disciplina en los alumnos visitantes de las Escuelas de Arquitectura de los Estados Unidos Mexicanos.

Palabras clave: prevención, seguridad, accidentalidad, estrategias docente, trabajo colaborativo.

\section{Teaching strategies for training of foreign students at the University of Burgos}

\begin{abstract}
There are many foreign students who choose our country to perfect their studies, doing exchanges with Spanish Universities. Annually, University of Burgos hosts students from different countries, specially from the United Mexican States. The agreements between University of Burgos and Mexican University of Baja California, San Luis Potosi, Chihuahua or Mexico City have enabled that many students of the Architecture School take subjects of Building Engineering Degree and Roads, Canals and Ports Engineer Degree. This paper develops a training strategy that professors of the subject of Prevention and Building Safety, which is part of the Building Engineering Degree, have been implemented with the aim to perfect the knowledge of this discipline in visitor's students from School of Architecture of the United Mexican States.

Keywords: prevention, safety, accidents, teaching strategies, collaborative work.

\section{Referencia normalizada}

RODRÍGUEZ SAIZ, Ángel y GUTIÉRREZ-GONZÁLEZ, Sara (2012) "Estrategias docentes para la formación de alumnos extranjeros en la Universidad de Burgos". Estudios sobre el Mensaje Periodístico, Vol. 19, Núm. especial marzo, págs.: 399-408. Madrid, Servicio de Publicaciones de la Universidad Complutense.
\end{abstract}

Sumario: 1. Introducción. 2. Metodología; 2.1. La formación general: objetivos y contenidos; 2.2. La formación específica complementaria: objetivos y contenidos. 3. Desarrollo. 4. Conclusiones. 5. Referencias bibliográficas. 


\section{Introducción}

La Universidad de Burgos tiene suscritos acuerdos con universidades de otros países para fomentar el intercambio de estudiantes de titulaciones afines, con el objetivo de conseguir una formación integral basada en experiencias docentes con distintas orientaciones programáticas. Al mismo tiempo, la movilidad interuniversitaria contribuye al enriquecimiento personal del alumno, ya que experimenta diferentes formas de organización social desde ámbitos culturales distintos al suyo. En los Estados Unidos Mexicanos estos intercambios son valorados positivamente, destacando las ventajas formativas obtenidas por los alumnos que han participado (Vázquez, 2011: Web).

En la Escuela Politécnica Superior de la Universidad de Burgos, este intercambio se ha visto incrementado de forma evidente en los últimos con alumnos procedentes de Universidades Mexicanas que participan en los denominados "Programas de Movilidad Estudiantil".

Estos alumnos cursan carreras técnicas relacionadas con la construcción de edificios y muestran un especial interés por cursar disciplinas específicas curriculares de las Titulaciones de Arquitectura Técnica, Ingeniería de Edificación e Ingeniería de Caminos Canales y Puertos.

Una de las asignaturas que eligen en su programación curricular es la de Prevención y Seguridad en la Construcción, que se imparte en el Tercer Curso de la Titulación de Ingeniería de Edificación. Para estos alumnos, la seguridad en las obras de construcción es una materia novedosa, ya que en sus Planes de Estudios no contienen asignaturas que estudien y traten de esta temática, por lo que encuentran atractiva la formación que se desarrolla en esta disciplina.

Para adaptar a los alumnos a la dinámica de estudio de nuestra Escuela y, en concreto, a las estrategias docentes que tenemos establecidas para el desarrollo de los contenidos, y al proceso de evaluación continua del Espacio Europeo de Educación Superior, los profesores de la Asignatura de Prevención y Seguridad en la Construcción hemos hecho un seguimiento personalizado de estos alumnos. Para ello, se han programado un conjunto de actividades alternativas, con el objetivo de conseguir un aprovechamiento académico adecuado en su experiencia docente durante su estancia en la Escuela Politécnica Superior de la Universidad de Burgos.

La experiencia docente se ha puesto en práctica con alumnos de los Estados Unidos Mexicanos, procedentes de diferentes Estados, que se encuentran participando el "Programa Alumnos de Intercambio Internacional", denominados en México "Programas de Movilidad Estudiantil"1, por lo que alumnos universitarios realizan estancias académicas en otras Instituciones de Educación Superior nacionales o del extranjero. Los créditos cursados son reconocidos si existe Convenio de Movilidad entre las Universidades colaboradoras.

1 Los programas de Movilidad Estudiantil ECEST (Espacio Común de la Educación Superior Tecnológica) son convocados anualmente por la Secretaría de Educación Pública de los Estados Unidos Mexicanos y tienen como objetivo promover la formación de alumnos mexicanos en universidades extranjeras. 
Los alumnos participantes son originarios de los Estados de Baja California Norte, Chihuahua, San Luis Potosí y México D.F. Todos ellos proceden de Facultades de Arquitectura y se encuentran matriculados en los últimos cursos de la carrera.

TABLA 1. Procedencia de los alumnos y Universidad de origen. (Elaboración propia)

\begin{tabular}{|c|c|c|c|c|}
\hline \multicolumn{5}{|c|}{ NÜMERO DE AIUMNOS Y PROCEI)ENCIA } \\
\hline ESTAIOO & (UNIVERSII)AI) & CAMPIS & FACULTAI) & ALAMNOS \\
\hline Bąj: California & Universidad Autónoma & Mexicali & Arquilectura & 4 \\
\hline San Luís Potosí & U/niversidad Autónoma & San Luis P'otosí & Arquitectura & 6 \\
\hline Chihuahua & Tecnológico de Monterrey & Chihuahua & Arquitectura & 1 \\
\hline México D.F. & Universidad Autónoma & México D.F. & Arquitectura & 1 \\
\hline
\end{tabular}

Las Universidades cuentan con diferentes Campus Universitarios dentro del mismo Estado. Cabe destacar el Instituto Tecnológico de Monterrey, que cuenta con varios Campus Universitarios en diferentes Estados en México y dispone de sedes en otros países.

La accidentalidad en la industria de la construcción en América Latina constituye una preocupación que se justifica por el número de trabajadores que pierden la vida en su trabajo y por la implicación que tiene este fenómeno en la economía de los países.

México es el país de América Latina en el que el Sector de la Construcción participa con mayor intensidad en su PIB, alcanzando el 6,8\% en 2011.

El Sector de la Construcción emplea anualmente un millón de trabajadores, registrándose un $8,92 \%$ de accidentes de trabajo y enfermedades profesionales, con una Tasa de Incidencia del 3,9\%. El número de fallecimientos por accidente alcanzó en 2010 un Índice de Incidencia del 2,32\%.

No obstante, aunque cada vez es mayor la concienciación de empresarios y trabajadores por la seguridad en las obras, son muchos los accidentes que todavía no se registran en las estadísticas del Instituto Mexicano de Seguridad Social.

Los alumnos mexicanos que eligen la asignatura de Prevención y Seguridad en la Construcción proceden principalmente de la Titulación de Arquitectura. En los Estados Unidos Mexicanos esta Titulación está orientada al diseño de edificios, las técnicas de representación, la funcionalidad de los espacios y su integración paisajística. El estudio urbanístico de las ciudades es otra disciplina que se fomenta en el estudio de la carrera, prestando especial importancia a la configuración del paisaje urbano, de acuerdo con las tipologías de ordenación del territorio y al respeto por el medio ambiente y el desarrollo sostenible.

La ejecución de los Proyectos Arquitectónicos es una competencia de los Ingenieros Civiles, profesionales que aplican las técnicas constructivas para plasmar en la realidad las ideas y creaciones de los Arquitectos. 
Al ser la seguridad una disciplina directamente relacionada con el proceso de ejecución de los proyectos, son los Ingenieros Civiles los que adquieren estas competencias durante su formación para el desarrollo y puesta en práctica efectiva de los principios de la acción preventiva que recoge la normativa mexicana.

La motivación que muestran los alumnos mexicanos para elegir la asignatura de Prevención y Seguridad en la Construcción se centra en la curiosidad por conocer una disciplina relacionada con su profesión, pero que no se contempla en los Planes de Estudio de Arquitectura que cursa en sus facultades. La inquietud es mayor aún cuando asocian la ejecución de los Proyectos Arquitectónicos al hecho puramente constructivo, es decir, a las técnicas y procedimientos de trabajo que se aplican para hacer realidad las creaciones que esbozan en sus diseños.

La prevención y seguridad no son materias ajenas a sus inquietudes como profesionales. Todos reconocen la necesidad de evitar que los trabajadores se accidenten en el desarrollo de sus trabajos, pero también todos coinciden que no hay una cultura preventiva. Aunque los Estados Unidos de México tienen una estructura jurídica y normativa semejante a la de España, el grado de cumplimiento presenta importantes contingencias y la sensibilidad real por la seguridad en las obras no es un valor de referencia en su país.

\section{Metodología}

Los alumnos mexicanos que cursan la Asignatura de Prevención y Seguridad en la Construcción, hacen un seguimiento de las actividades propuestas por los profesores responsables semejante al de los alumnos nacionales. En este sentido, el alumno visitante se incorpora al proceso de aprendizaje con los mismos requerimientos exigidos al resto de alumno nacionales.

El propósito de la "formación añadida" radica en aprovechar una formación sólida en prevención de riesgos laborales, adaptándola a las singularidades del marco normativo jurídico-técnico de los Estados Unidos Mexicanos. Todas las estrategias formativas desarrolladas en las actividades propuestas se aprovechan para, de forma paralela, establecer un parangón con la realidad de su propio país.

\subsection{La formación general: objetivos y contenidos}

Para el seguimiento de la asignatura de Prevención y Seguridad en la Construcción, los alumnos deben configurar su "Portafolio personal de trabajo". Este recurso organizativo permite organizar el conjunto de actividades docentes que configuran la estructura curricular de la asignatura y que, de una forma descriptiva, se refieren a continuación:

a) Asistencia presencial a las clases magistrales, donde se exponen los contenidos teóricos de la asignatura, de acuerdo con el Programa desarrollado en la Guía Docente de la asignatura.

b) Trabajos de aula, significando las actividades individuales, de grupo, trabajo cooperativo, estudio de casos, conferencias etc., de acuerdo con los postulados programáticos del Espacio Europeo de Educación Superior.

c) Asistencia presencial a clases prácticas, donde se desarrolla la casuística constructiva en relación con la prevención y seguridad de los procesos constructi- 
vos, las técnicas de trabajo y el análisis de contingencias potenciales en las diferentes actividades de obra.

d) Talleres de Aula donde se elabora un Estudio de Seguridad o Plan de Seguridad y Salud, análisis de casos prácticos concretos e informes de seguridad sobre situaciones críticas que pueden acontecer en las obras de edificación.

Los alumnos suelen trabajar juntos, ya que comparten una misma organización curricular y las asignaturas en las que se matriculan, en la mayor parte de los casos, son las mismas.

Finalizado el proceso de enseñanza-aprendizaje, se procede a valorar su participación en las actividades propuestas de acuerdo con el modelo de "evaluación continua", siguiendo los postulados del Espacio Europeo de Educación Superior, método que permite la retroalimentación del sistema de enseñanza-aprendizaje, resultando una herramienta muy útil para el profesor (Delgado et al. 2006: web) . Esta valoración se configura atendiendo a las siguientes referencias:

a) Superación de las pruebas de conocimiento de los contenidos desarrollados en las "clases teóricas y prácticas".

b) Desarrollo de todas las actividades propuestas como "trabajo personal" mediante la presentación de su "portafolio" personal.

c) Entrega de un Estudio de Seguridad o Plan de Seguridad y Salud propuesto por los tutores, y superación del nivel de calidad exigido para el mismo.

La valoración de las actividades realizadas y del grado de excelencia alcanzado en cada una de ellas constituye la "nota final", que refiere el nivel de aprovechamiento obtenido por el alumno en el conjunto de la asignatura. Esta nota parecerá en el Expediente Académico que el Servicio de Relaciones Internacionales trasladará a su Universidad de origen.

\subsection{La formación específica complementaria: objetivos y contenidos}

Con el ánimo de premiar la estancia de los alumnos mexicanos en la Universidad de Burgos, los profesores de la asignatura de Prevención y Seguridad en la Construcción posibilitamos el acceso a un "Cerificado de Colaboración", mediante su participación en una "formación complementaria", con actividades orientadas al ámbito de referencia en el que van a ejercer profesionalmente, es decir, el marco jurídico-técnico de los Estados Unidos Mexicanos.

La participación del alumno con aprovechamiento en estas actividades tiene como recompensa el incremento en un grado de la nota final alcanzada en la evaluación ordinaria de la asignatura de Prevención y Seguridad en la Construcción, descrita anteriormente.

El Certificado de Colaboración es un documento que se facilita al alumno y en el que se recoge, además del seguimiento de la asignatura, un reconocimiento de su participación en una formación específica con actividades relacionadas con el estudio y conocimiento técnico y jurídico de la normativa de referencia en los Estados Unidos Mexicanos, en materia preventiva y de seguridad en las obras de edificación. 
Los alumnos son informados cuando se incorporan a nuestra asignatura de estas actividades de colaboración, a las que el alumno puede adherirse voluntariamente. Los alumnos que no participan, reciben la nota final de la evaluación ordinaria en su Expediente Académico.

GRÁFICO 1. Evaluación final del alumnado. (Elaboración propia)

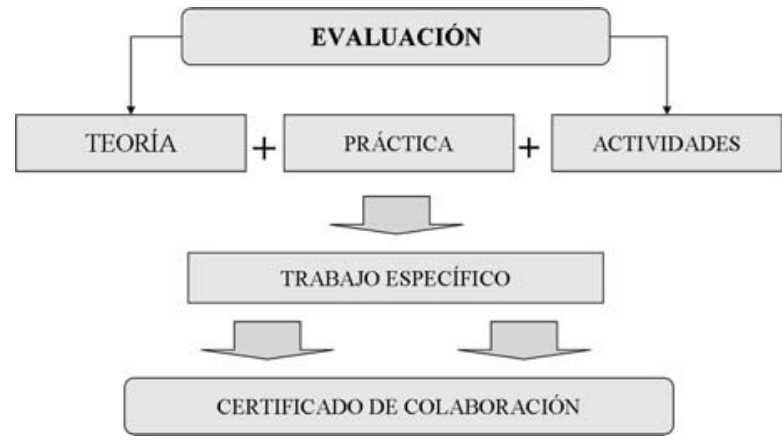

La propuesta ha contado siempre con el interés de los alumnos, ya que les permite completar su formación adquiriendo un conjunto de competencias y habilidades en una materia que no se recoge en los Planes de Estudio de las Facultades de Arquitectura de México.

Los objetivos que persigue esta formación complementaria son los siguientes:

a) Adquirir un conocimiento profesional sobre la problemática de la accidentalidad en la construcción y, específicamente, de la prevención y seguridad en la ejecución de los edificios y construcciones que proyecten.

b) Ser consciente de la responsabilidad que adquieren como proyectistas en el seguimiento de los procesos de ejecución de las edificaciones, siendo conscientes de que la "ética profesional" debe estar presente en todas sus actuaciones.

c) Adquirir un conocimiento específico sobre el "Marco Jurídico y Técnico" de los Estados Unidos Mexicanos en materia preventiva.

d) Adquirir habilidades para la puesta en práctica de la "cultura preventiva", con el propósito de prevenir cualquier contingencia potencial y futura que pueda afectar a la integridad física o psíquica de los trabajadores de la construcción.

Con estas actividades, los alumnos van a adquirir una visión más objetiva sobre la problemática de la accidentalidad en las obras de construcción.

Las actividades que se proponen se refieren a la búsqueda de información sobre el Marco Normativo que organiza y fundamenta la prevención y seguridad en las obras de construcción en los Estados Unidos de México.

Las actividades que se proponen se refieren en los siguientes apartados:

a) Referencias normativas de "carácter general" tales como Leyes, Reglamentos, Decretos, etc., es decir, un análisis sucinto del Marco Normativo que regula las relaciones de trabajo en los Estados Unidos Mexicanos.

b) Referencias normativas de "carácter específico" que regulan la seguridad en la actividad laboral, diferenciando las Leyes Federales, Estatales o de los municipios. 
c) Estadísticas de accidentalidad laboral en México y, específicamente, en el Sector de la Construcción.

d) Estudio de los "organismos institucionales" con responsabilidad en material laboral preventiva, con especial referencia al Sistema de Inspección y a las sanciones en caso de incumplimientos en materia preventiva.

e) Sistema organizativo de la prevención y seguridad en las obras, indicando las acciones que deben realizar las empresas para prevenir los riesgos derivados del trabajo en las obras de construcción.

f) Documentos que deben disponerse para realizar las labores preventivas, tales como evaluaciones de riesgos, formación e información a los trabajadores, documentación de obra, Servicios de Prevención, etc.

g) Estudio comparativo de la Legislación Española y Comunitaria con la de los Estados Unidos Mexicanos.

\section{Desarrollo}

Para un desarrollo adecuado de las actividades descritas, los profesores de la asignatura han seguido un proceso de formativo específico en técnicas y recursos utilizados en el proceso de enseñanza-aprendizaje del Espacio Europeo de Educación Superior en el Instituto de Formación e Innovación Educativa de la Universidad de Burgos (IFIE), obteniendo el Certificado de Estudios Europeos que emite este organismo'.

Para la elaboración del trabajo se puede elegir libremente la modalidad de trabajo individual o en grupo. Cuando son varios los alumnos, el trabajo se reparte y cada uno desarrolla uno o varios apartados del mismo. No se pide un trabajo al uso (montones de folios escritos y encuadernados), sino una exposición fundamentada con referencias sustentadas en una búsqueda bibliográfica, en documentos oficiales, en estadísticas, o en otros trabajos.

El trabajo realizado, bien individualmente o en grupo, se debe exponer al resto de compañeros mediante una puesta en común de la información recogida. Para ello disponemos de una sala de trabajo de dimensiones reducidas, dotada de proyector y ordenador, con un ambiente que contribuye a la participación de todos los alumnos.

Aunque la técnica expositiva es muy formalista, los Tutores facilitamos el debate de las propuestas para comprobar el conocimiento del alumno, la calidad de la información recabada y el grado de interacción con el resto de compañeros. El trabajo colaborativo en pequeños grupos, poniendo en común el trabajo desarrollado individualmente, incentiva la cooperación entre iguales y proporcionan una mayor atracción interpersonal (Barrachina et al., 2008: 123-124).

La evaluación de los trabajos no es cuantitativa, sino una valoración general sobre la información aportada, las habilidades de comunicación de los alumnos y el interés

2 El Certificado de Estudios Europeos es un documento expedido por el Instituto de Formación del Profesorado e Innovación Educativa de la Universidad de Burgos que acredita una formación mínima de 200 horas en técnicas de trabajo aplicables a la metodología de enseñanza del Espacio Europeo de Educación Superior. 
mostrado durante su desarrollo. De igual forma, la participación en los debates es una referencia también para la valoración de las habilidades adquiridas.

Los alumnos que han hecho seguimiento de las actividades del Programa de Colaboración reciben un documento acreditativo que, además de recoger el grado de aprovechamiento en las actividades comunes de la asignatura de Prevención y Seguridad en la Construcción, también describe los trabajos realizados en la "formación específica complementaria" para los alumnos del Programa de Movilidad Estudiantil México-España.

El documento es propuesto por los profesores-tutores a la Dirección del Departamento de Construcciones Arquitectónicas e Ingeniería de la Construcción y del Terreno, para su firma.

El "Certificado de Colaboración" es una justa recompensa a su esfuerzo y supone un reconocimiento a su participación en las actividades extracurriculares de la Asignatura de Prevención y Seguridad en la Construcción. Además, dependiendo del grado de aprovechamiento alcanzado, los Profesores-Tutores incrementan en un grado la nota alcanzada en la "evaluación ordinaria", trasladándose ésta al Expediente Personal de cada alumno, gestionado el Servicio de Relaciones Internacionales e la Universidad de Burgos.

Ilustración 1. Certificado de Colaboración. (Elaboración Propia)

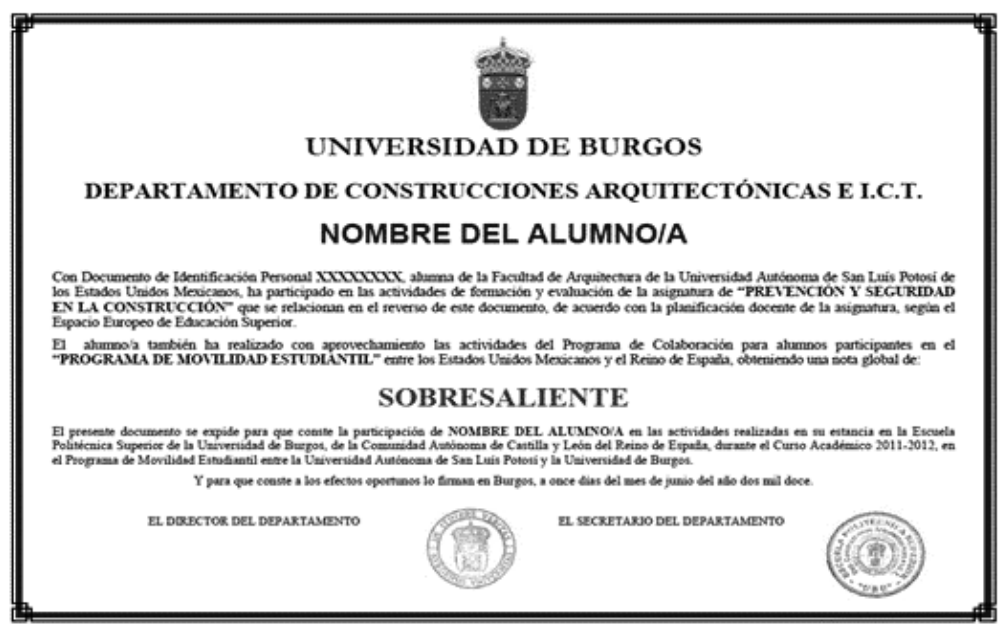

\section{Conclusiones}

Los profesores de la asignatura de Prevención y Seguridad en la Construcción consideramos que la actividad que hemos desarrollado con los alumnos del "Programa Alumnos de Intercambio Internacional", denominados en México "Programas de Movilidad Estudiantil", es muy positiva, ya que se incentiva el interés de los alumnos por adquirir conocimientos en una materia específica, como es la Prevención y Seguridad en las Obras de Construcción, referida al ámbito técnico-jurídico de los Estados Unidos Mexicanos, que es donde van a poner en práctica las habilidades profesionales adquiridas en su formación como Arquitectos. 
Supone un trabajo añadido al desarrollado en la propia asignatura, pero sirve para poner en práctica técnicas educativas de enseñanza-aprendizaje con un grupo reducido de alumnos, circunstancia que no se da en la asignatura oficial de Prevención y Seguridad en la Construcción, del Programa de Estudios de la Titulación de Ingeniería de Edificación, ya que, la cantidad de alumnos matriculados impide la puesta en práctica de técnicas específicas de enseñanza-aprendizaje del Espacio Europeo de Educación Superior.

Además de los procesos de enseñanza-aprendizaje puestos en marcha, los profesores tratamos de cumplir nuestra función de mediadores en el proceso de aprendizaje, trasladando a los alumnos los "valores humanos y sociales" que deben fundamentar las actuaciones profesionales (Cerrillo, 2003:62-63). Desde esta perspectiva, la respuesta de los alumnos ha sido muy positiva, ya que se sienten sensibilizados con la problemática de la accidentalidad y de las condiciones de trabajo.

Desde el punto de vista personal, se agradece la participación de los alumnos, el respeto y consideración que tienen con los profesores y la educación que muestran con el resto de compañeros.

La valoración final es positiva, enriquece a los propios profesores y facilita la puesta en valor de la formación adquirida en el Instituto de Formación e Innovación Educativa para el nuevo Espacio Europeo de Educación Superior.

\section{Referencias bibliográficas}

ANDRÉU BARRACHINA, Llorenç; SANZ TORRENT, Mónica y SERRAT I SELLABONA, Elisabeth (2008): "Una propuesta de renovación metodológica en el marco del Espacio Europeo de Enseñanza Superior: los pequeños grupos de investigación cooperativos". Revista Electrónica Interuniversitaria de Formación del Profesorado REIFOP, 12 (3), pp. 123-124. http://www.aufop.com/aufop/uploaded_files/articulos/1254437275.pdf [fecha de consulta: 5 de noviembre de 2012]

CERILLO MARTÍN, María del Rosario (2003): "Educar en valores: misión del profesor". Tendencias Pedagógicas, $\mathrm{n}^{\circ}$ 8. Departamento de Didáctica y Teoría de la Educación de la Universidad Autónoma de Madrid, pp. 59-68.

DELGADO GARCÍA, Ana María y OLIVER CUELLO, Rafael (2006): "La evaluación continua en un nuevo escenario docente". Revista de Universidad y Sociedad del Conocimiento. Vol. 3, $\mathrm{n}^{\mathrm{o}}$ 1. http://www.uoc.edu/rusc/3/1/dt/esp/delgado_oliver.pdf [fecha de consulta: 5 de noviembre de 2012]

VÁZQUEZ Y ROMERO, Lázaro (2011): "Ventajas y desventajas de la movilidad interuniversitaria". Revista Electrónica del Programa de Estudios Universitarios Comparados, $\mathrm{n}^{\circ}$ 13. http://www.peu.buap.mx/Revista_13/numeros_anteriores.html [fecha de consulta: 5 de noviembre de 2012] 


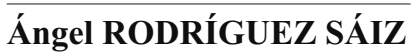

Doctor Ingeniero Organización Industrial, Licenciado en Derecho y Arquitecto Técnico Profesor Titular de Universidad adscrito al Departamento de Construcciones Arquitectónicas e Ingeniería de la Construcción y del Terreno de la Universidad de Burgos.

Director del Departamento de Construcciones Arquitectónicas e Ingeniería de la Construcción y del Terreno de la Universidad de Burgos.

arsaizmc@ubu.es

\section{Sara GUTIÉRREZ GONZÁLEZ}

Doctora Ingeniero Organización Industrial y Arquitecto Técnico

Profesora Ayudante adscrita al Departamento de Construcciones Arquitectónicas e Ingeniería de la Construcción y del Terreno de la Universidad de Burgos.

sggonzalez@ubu.es 